\title{
Thoracic Endovascular Aortic Repair With Left Subclavian Artery Coverage Is Associated With a High 30-Day Stroke Incidence With or Without Concomitant Revascularization
}

\author{
Rens R. B. Varkevisser, BS ${ }^{1,2}$ iD, Nicholas J. Swerdlow, MD', \\ Livia E. V. M. de Guerre, MD', Kirsten Dansey, MD', Chun Li, MD', \\ Patric Liang, MD', Christopher A. Latz, MD', \\ Mathijs T. Carvalho Mota, BS', Hence J. M. Verhagen, MD, PhD², \\ and Marc L. Schermerhorn, MD'
}

\begin{abstract}
Purpose: To evaluate the perioperative stroke incidence following thoracic endovascular aortic repair (TEVAR) with differing left subclavian artery (LSA) coverage and revascularization approaches in a real-world setting of a nationwide clinical registry. Materials and Methods: The National Surgical Quality Improvement Program registry was interrogated from 2005 to 2017 to identify all nonemergent TEVAR and/or open LSA revascularization procedures. In this time frame, 2346 TEVAR cases met the selection criteria for analysis. The 30-day stroke incidence was compared between patients undergoing TEVAR with $(n=888)$ vs without $(n=1458)$ LSA coverage, for those with $(n=228)$ vs without $(n=660)$ concomitant LSA revascularization among those with coverage, and following isolated LSA revascularization for occlusive disease $(n=768)$. Multivariable logistic regression was employed for risk-adjusted analyses and to identify factors associated with stroke following TEVAR. Results of the regression analyses are presented as the adjusted odds ratio (OR) with $95 \%$ confidence interval $(\mathrm{Cl})$. Results: The stroke incidence was $2.3 \%$ following TEVAR without vs $5.2 \%$ with LSA coverage $(p<0.001)$. In TEVARs with LSA coverage, the stroke incidence was $7.5 \%$ when the LSA was concomitantly revascularized and $4.4 \%$ without concomitant revascularization, while stroke occurred in $0.5 \%$ of isolated LSA revascularizations. Of 33 TEVAR patients experiencing a perioperative stroke, 8 (24\%) died within 30 days. LSA coverage was associated with stroke both with concomitant revascularization $(\mathrm{OR} 4.0,95 \% \mathrm{Cl} 2.2$ to $7.5, \mathrm{p}<0.00 \mathrm{I})$ and without concomitant revascularization (OR 2.2, 95\% $\mathrm{Cl}$ I.3 to 3.8, $\mathrm{p}=0.002)$. Other preoperative factors associated with stroke were dyspnea $(\mathrm{OR} I .8,95 \% \mathrm{Cl} I .1$ to $3.0, p=0.014$ ), renal dysfunction (OR $2.2,95 \% \mathrm{Cl}$ I.0 to $3.8, p=0.049$ ), and international normalized ratio $\geq 2.0$ (OR 3.6 , $95 \% \mathrm{Cl} 1.0$ to 13, $\mathrm{p}=0.045$ ). Conclusion: Stroke following TEVAR with LSA coverage occurs frequently in the real-world setting, and concurrent LSA revascularization was not associated with a lower stroke incidence.
\end{abstract}

\section{Keywords}

endograft, left subclavian artery, LSA coverage, registry, revascularization, risk factors, stent-graft, stroke, thoracic aortic aneurysm, thoracic dissection, thoracic endovascular aortic repair

\section{Introduction}

Thoracic endovascular aortic repair (TEVAR) is a minimally invasive alternative to open surgery for a variety of thoracic aortic pathologies, including thoracic aortic aneurysms (TAA) and dissections. By avoiding a thoracotomy incision and aortic cross clamping, TEVAR has favorable perioperative outcomes compared to open surgical repair. ${ }^{1}$
Nevertheless, TEVAR has not reduced the risk of perioperative stroke compared to open surgery. ${ }^{2}$

An important consideration for TEVAR is adequate proximal sealing of the graft to ensure successful exclusion of the aortic pathology. Up to $40 \%$ of all patients undergoing TEVAR have lesions that extend to or involve the origin of the left subclavian artery (LSA), which necessitates coverage of the LSA for adequate stent-graft seal. ${ }^{3}$ LSA coverage 
leads to reduced or obstructed blood flow to the left vertebral artery, which theoretically can provoke cerebrovascular ischemia and stroke. Additionally, coverage of the more proximal aortic arch requires delivery of bulky stent-grafts across the origin of the arch vessels, potentially leading to embolization into the cerebral circulation. In an attempt to reduce the stroke incidence following TEVAR with coverage of the LSA, the Society of Vascular Surgery guidelines recommend routine LSA revascularization in TEVAR procedures that necessitate coverage of the LSA, though they do acknowledge that the current evidence for this recommendation is poor. ${ }^{4}$ Two recent meta-analyses demonstrated that LSA revascularization did not lower the stroke incidence after TEVAR, with pooled stroke rates of $4.7 \%$ and $7.8 \%$ for coverage without revascularization and $4.1 \%$ and $5.8 \%$ for those with a revascularization procedure. ${ }^{5,6}$ However, data included in these studies may not be fully generalizable due to publication bias, raising the question as to what are the real-world outcomes following TEVAR. ${ }^{5,6}$

The purpose of the current study was to report the realworld perioperative stroke incidence following TEVAR with and without coverage of the LSA using data from a nationwide clinical registry. Additionally, the stroke incidence for TEVAR procedures with LSA coverage with and without concomitant LSA revascularization was examined and compared with isolated LSA revascularizations for occlusive diseases alone.

\section{Materials and Methods}

\section{Data Source}

Data were extracted from the American College of Surgeons National Surgical Quality Improvement Program (NSQIP) registry (www.facs.org/quality-programs/acs-nsqip), which in 2005 began collecting data from surgical procedures performed in over 700 participating hospitals. The NSQIP registry collects granular data, recorded in over 270 variables, including patient demographics, comorbidities, and procedural characteristics, as well as outcomes up to 30 days after the index procedure, with minimal missing data. Data are collected by trained clinical nurses and data abstractors, and the registry has been validated previously. ${ }^{7,8}$ The NSQIP uses a random sampling protocol to gather information, so the number of patients in this study does not necessarily represent all cases performed in the participating centers. In addition, center and surgeon identifiers are not provided by the registry. As the NSQIP registry uses deidentified data, the Institutional Review Board of the Beth Israel Deaconess Medical Center approved this study and waived the requirement of an informed consent.

\section{Patient Cohort}

The database was interrogated up to 2017 to identify all TEVAR procedures of the descending thoracic aorta using the primary or concomitant (under the same anesthesia) Current Procedural Terminology (CPT) codes: 33880, 75956, 0033T, and 0038T for TEVAR with LSA coverage and 33881, 75957, 0034T, and 0039T for TEVAR without LSA coverage. The NSQIP does not capture proximal landing zones, so the data were stratified only on coverage of the LSA during TEVAR. Primary or concomitant open LSA revascularization was identified by CPT codes for such procedures (Supplementary Table 1; available in the online version of the article). Based on these identified procedures, patients were categorized as undergoing (1) TEVAR without LSA coverage, (2) TEVAR with LSA coverage without concomitant revascularization, (3) TEVAR with LSA coverage and concomitant revascularization, and (4) isolated LSA revascularization (without TEVAR). Isolated LSA revascularization procedures were included only if the primary diagnosis code (International Classification of Disease, 9th or 10th revision) was occlusive disease (Supplementary Table 2).

Patients were excluded if treated for ruptured TAA or had procedures coded as emergent $(\mathrm{n}=1086)$, as well as TEVAR performed for primary indications other than TAA or thoracic aortic dissection $(n=1239)$. In addition, patients who were intubated preoperatively $(\mathrm{n}=29)$ were eliminated as this likely represented trauma patients who may have other reasons for stroke. Another 49 cases were excluded because the CPT codes for the primary procedure were unlikely primary TEVAR or LSA revascularization; a full list of excluded primary CPT codes is presented in Supplementary Table 3. This culling left 2346 patients undergoing TEVAR for the analysis (Figure 1). Of these, $1458(62 \%)$ patients (median age 72 years; 804 men) had no LSA coverage while 888 (median age 69 years; 515 men) did; among the latter, $228(26 \%)$ underwent concomitant LSA revascularization. There were 768 patients (median age 64 years; 501 women)

'Department of Surgery, Division of Vascular and Endovascular Surgery, Beth Israel Deaconess Medical Center, Harvard Medical School, Boston, MA, USA

${ }^{2}$ Department of Vascular Surgery, Erasmus University Medical Center Rotterdam, the Netherlands

${ }^{3}$ Division of Vascular and Endovascular Surgery, Massachusetts General Hospital, Boston, MA, USA

\section{Corresponding Author:}

Marc L. Schermerhorn, MD, FACS, Department of Surgery, Division of Vascular and Endovascular Surgery, Beth Israel Deaconess Medical Center, I I0 Francis Street, Suite 5B, Boston, MA 02215, USA.

Email: mscherm@bidmc.harvard.edu 


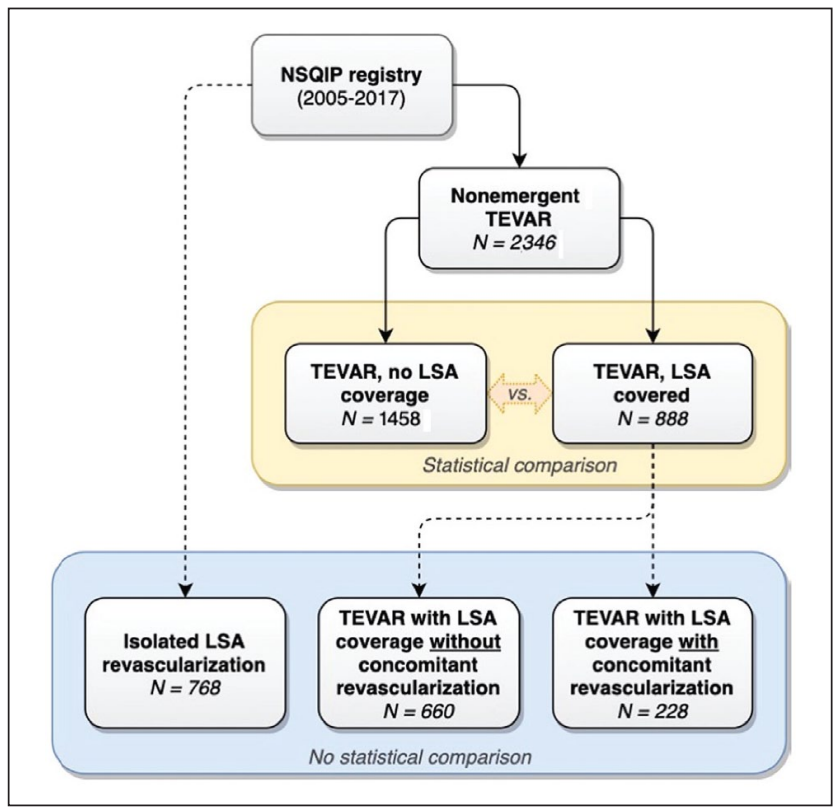

Figure I. Flow diagram of patients included in the study. LSA, left subclavian artery; NSQIP, National Surgical Quality Improvement Program; TEVAR, thoracic endovascular aortic repair.

undergoing isolated LSA revascularization for occlusive disease. Baseline demographics and comorbidities are presented in Table 1.

Outcomes were compared between TEVAR procedures with vs without LSA coverage, TEVAR with LSA coverage with or without concomitant LSA revascularization, and isolated LSA revascularization for occlusive disease (Figure 1). Since the NSQIP does not capture prior procedures or planned reoperations, it is likely that some of the patients in the TEVAR with LSA coverage without concomitant revascularization group underwent staged procedures (prior or subsequent revascularization; not under the same anesthesia). Therefore, this group likely comprised patients without any LSA revascularization and patients with staged revascularization. This heterogeneity compromised valid statistical testing between TEVAR with coverage with vs without concomitant LSA revascularization. However, since this does not apply for the comparison to TEVAR without LSA involvement, both LSA covered TEVAR groups (with or without concomitant revascularization) were not compared with the no LSA involvement group in multivariable models.

\section{Definitions and Outcome Measures}

Underweight and obesity were defined according to the body mass index as $\leq 18.5$ or $>30 \mathrm{~kg} / \mathrm{m}^{2}$, respectively. Renal dysfunction was defined as an estimated glomerular filtration rate $(\mathrm{eGFR})<60 \mathrm{~mL} / \mathrm{min} / 1.72 \mathrm{~m}^{2}$ or dialysis; severe renal dysfunction was an eGFR $<30$ or preoperative dialysis (regardless of eGFR). Estimated eGFR was calculated according to the Chronic Kidney Disease Epidemiology Collaboration equation. ${ }^{9}$ The preoperative international normalized ratio (INR) was derived from the diagnostic blood test closest to the index procedure (only within 90 days prior to the procedure). Brachial artery access was identified by concurrent CPT code 34834 .

The primary outcome of this study was any stroke within 30 days from the index procedure. Stroke was defined by the NSQIP as motor, sensory, or cognitive dysfunction persisting for $\geq 24$ hours and clinical suspicion of stroke. Secondary outcomes included 30-day overall mortality and death following a stroke. This analysis also included major complications defined as captured in the registry as unplanned reoperation, myocardial infarction, cardiac arrest, reintubation, failure to wean from the ventilator within 48 hours after the procedure, pulmonary embolism, renal failure requiring dialysis, sepsis, or septic shock.

\section{Statistical Analysis}

Categorical variables are presented as counts and percentages and continuous variables as median and interquartile range (IQR Q1, Q3). Baseline characteristics and outcomes were compared and tested for differences using the Pearson chi-square test or Fisher exact test for categorical variables and the Mann-Whitney $U$ tests for continuous variables.

Multivariable logistic regression was used to identify the risk-adjusted association between the primary procedure and postoperative stroke. Covariates for these models were selected a priori and included surgery indication (TAA vs dissection), age, sex, smoking status, insulin-dependent diabetes mellitus, chronic obstructive pulmonary disease (COPD), congestive heart failure, bleeding disorders, severe preoperative renal dysfunction, preoperative dyspnea, brachial artery access, partial thromboplastin time (PPT) $<25$ seconds, and INR $\geq 2.0$. Last, factors associated with stroke following TEVAR were identified by creating a model with stroke as a dependent variable and all potential associated factors available in the database as independent variables. Stepwise backward selection was employed until all included factors reached significant levels. Outcome variables had no missing data, but some other variables with $>2 \%$ missing data were race (7.4\%), INR (13\%), and PTT (23\%). Indicator variables were created in the multivariable models for INR $>2$ and PTT $>25$ seconds to maintain statistical power, resulting in $98.4 \%$ complete cases in those models. Results of the regression modeling are presented as the odds ratio (OR) with $95 \%$ confidence interval (CI).

All tests were 2-sided; $\mathrm{p}<0.05$ indicated a statistically significant difference. All analyses were performed using 
Table I. Baseline Characteristics of the Patients Undergoing TEVAR and Isolated LSA Revascularization. ${ }^{a}$

\begin{tabular}{|c|c|c|c|c|}
\hline \multirow[b]{2}{*}{ Characteristic } & \multicolumn{3}{|c|}{ TEVAR } & \multirow{2}{*}{$\begin{array}{c}\text { Isolated LSA } \\
\text { Revascularization } \\
\qquad(n=768)\end{array}$} \\
\hline & $\begin{array}{l}\text { No LSA Coverage } \\
\qquad(n=1458)\end{array}$ & $\begin{array}{l}\text { LSA Covered } \\
\quad(n=888)\end{array}$ & $P$ & \\
\hline Age, y & $72(63,79)$ & $69(60,77)$ & $<0.001$ & $64(56,73)$ \\
\hline Female sex & $654 / 1458$ (44.9) & $373 / 888(42.0)$ & 0.18 & $501 / 758(65.2)$ \\
\hline White race & 1094/I368 (80.0) & $598 / 804(74.4)$ & 0.002 & 662/724 (91.4) \\
\hline Black race & $230 / 1368(16.8)$ & I56/804 (19.4) & 0.13 & $39 / 724(5.4)$ \\
\hline Current smoker & $462 / 1458(31.7)$ & $262 / 888(29.5)$ & 0.27 & $363 / 768(47.3)$ \\
\hline Underweight $(\mathrm{BMI}<\mathrm{I8.5})$ & $48 / 1430(3.4)$ & $24 / 870(2.8)$ & 0.42 & $22 / 762(2.9)$ \\
\hline Obese $(\mathrm{BMI}>30)$ & $450 / 1430(31.4)$ & $287 / 870(33.0)$ & 0.45 & $237 / 762(31.1)$ \\
\hline IDDM & $53 / 1458(3.6)$ & $29 / 888(3.3)$ & 0.64 & $75 / 768(9.8)$ \\
\hline Hypertension & I290/I458 (88.5) & 777/888 (87.5) & 0.48 & $582 / 768(75.8)$ \\
\hline Congestive heart failure & $27 / 1458$ (I.9) & $25 / 888(2.8)$ & 0.12 & $19 / 768(2.5)$ \\
\hline Dyspnea & $37 \mathrm{I} / \mathrm{I} 458(25.4)$ & I80/888 (20.3) & 0.004 & I72/768 (22.4) \\
\hline COPD & $290 / 1458$ (19.9) & I29/888 (I4.5) & 0.001 & 139/768 (18.1) \\
\hline Any renal dysfunction & $601 / 1432(42.0)$ & 297/877 (33.9) & $<0.001$ & $188 / 740(25.4)$ \\
\hline Severe renal dysfunction & $110 / 1432(7.7)$ & $65 / 877(7.4)$ & 0.81 & $22 / 740(3.0)$ \\
\hline TAA (vs dissection) & I I40/I458 (78.2) & 622/888 (70.0) & $<0.001$ & NA \\
\hline Brachial artery access & I4/I 458 (I.0) & $36 / 888(4.1)$ & $<0.001$ & NA \\
\hline INR $\geq 2.0$ & I4/I266 (I.I) & I3/768 (I.7) & 0.26 & $3 / 567(0.5)$ \\
\hline
\end{tabular}

Abbreviations: BMI, body mass index, $\mathrm{kg} / \mathrm{m}^{2}$; COPD, chronic obstructive pulmonary disease; IDDM, insulin-dependent diabetes mellitus; INR, international normalized ratio; LSA, left subclavian artery; NA, not applicable; TAA, thoracic aortic aneurysm; TEVAR, thoracic endovascular aortic repair.

${ }^{a}$ Continuous data are presented as the median (interquartile range QI, Q3); categorical data are given as the number (percentage).

Table 2. Univariable Analysis of Outcomes Following TEVAR. ${ }^{\mathrm{a}}$

\begin{tabular}{|c|c|c|c|}
\hline & No LSA Coverage $(n=\mid 458)$ & LSA Coverage $(n=888)$ & $P$ \\
\hline 30-Day mortality & $41(2.8)$ & $42(4.7)$ & 0.015 \\
\hline Stroke & $33(2.3)$ & $46(5.2)$ & $<0.001$ \\
\hline Died after stroke & $8 / 33(24.2)$ & I I/46 (23.9) & 0.97 \\
\hline Major complication & $162(11.2)$ & $15 \mid(\mid 7.0)$ & $<0.001$ \\
\hline
\end{tabular}

Abbreviations: LSA, left subclavian artery; TEVAR, thoracic endovascular aortic repair.

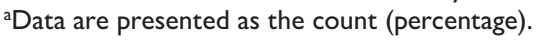

Stata software (version 15.1; StataCorp, College Station, TX, USA).

\section{Results}

\section{Group Comparisons}

Patients undergoing TEVAR with LSA coverage were younger compared to those without LSA coverage $(\mathrm{p}<0.001$; Table 1). There was no difference in the proportion of female patients between patients with vs without LSA coverage $(\mathrm{p}=0.18)$, while patients with LSA coverage were less often white $(\mathrm{p}=0.002)$, with preoperative dyspnea $(\mathrm{p}=0.004)$, COPD $(p=0.001)$, or renal dysfunction $(\mathrm{p}<0.001)$. The proportion of patients undergoing TEVAR for TAA (vs dissection) was lower for TEVAR with LSA coverage $(\mathrm{p}<0.001)$, whereas brachial access was established more often in patients with LSA involvement $(\mathrm{p}<0.001)$. Patients undergoing isolated LSA revascularization were more often female and white; slightly fewer than half were active smokers. Furthermore, a third were obese, a quarter had renal dysfunction, and 1 in 10 had insulin-dependent diabetes.

\section{Outcomes}

Stroke occurred in 46 patients $(5.2 \%)$ undergoing TEVAR with LSA coverage and in 33 patients $(2.3 \%)$ following TEVAR without LSA coverage $(\mathrm{p}<0.001$; Table 2$)$. Of all TEVAR patients experiencing a stroke within 30 days postoperatively, $24 \%$ died within that same period. Thirtyday mortality was $4.7 \%$ in patients with coverage and $2.8 \%$ in patients without $(\mathrm{p}=0.015)$. Furthermore, major 
Table 3. Adjusted Multivariable Association Between Primary Procedure and Stroke. ${ }^{a}$

\begin{tabular}{lr}
\hline Coverage (vs No Coverage as Reference) & $2.7(\mathrm{I} .7$ to 4.3$) \mathrm{p}<0.00 \mathrm{I}$ \\
LSA coverage & $2.2(\mathrm{I} .3$ to 3.8$) \mathrm{p}=0.002$ \\
Without concomitant revascularization $^{\mathrm{b}}$ & $4.0(2.2$ to 7.5$) \mathrm{p}<0.00 \mathrm{I}$ \\
With concomitant revascularization & 4.02
\end{tabular}

Abbreviation: LSA, left subclavian artery.

${ }^{\mathrm{a}} \mathrm{D}$ ata are presented as the odds ratio ( $95 \%$ confidence interval).

${ }^{\mathrm{b}}$ This does not account for stroke risk associated with prior or subsequent revascularization.

Table 4. Outcomes for TEVAR Patients With LSA Coverage and Isolated LSA Revascularization for Occlusive Disease. ${ }^{a}$

\begin{tabular}{lccc}
\hline & \multicolumn{2}{c}{ TEVAR With LSA Coverage } & Isolated LSA \\
\cline { 2 - 3 } & $\begin{array}{c}\text { Concomitant } \\
\text { Revascularization }(\mathrm{n}=660)\end{array}$ & $\begin{array}{c}\text { No Concomitant } \\
\text { Revascularization }(\mathrm{n}=228)\end{array}$ & $\begin{array}{c}\text { Revascularization } \\
(\mathrm{n}=768)\end{array}$ \\
\hline 30-Day mortality & $29(4.4)$ & $13(5.7)$ & $4(0.5)$ \\
Stroke & $29(4.4)$ & $17(7.5)$ & $4(0.5)$ \\
$\quad$ Died after stroke & $7 / 29(24.1)$ & $4 / 13(30.8)$ & $1 / 4(25.0)$ \\
Major complication & $101(15.3)$ & $50(21.9)$ & $43(5.6)$ \\
\hline
\end{tabular}

Abbreviations: LSA, left subclavian artery; TEVAR, thoracic endovascular aortic repair.

${ }^{a}$ Data are presented as the count (percentage).

complications other than stroke occurred more frequently following LSA coverage $(17 \%$ vs $11 \%, \mathrm{p}<0.001)$. There was no difference in the stroke rate between TEVAR for TAA vs thoracic dissection (no LSA coverage $2.4 \%$ vs $2.5 \% ; \mathrm{p}=0.73$; LSA coverage $5.6 \%$ vs $4.1 \%, \mathrm{p}=0.36$ ). After risk adjustment, LSA coverage was associated with higher odds of stroke (OR 2.7, 95\% CI 1.7 to 4.3, p $<0.001$; Table 3) compared to TEVAR without LSA involvement.

Perioperative stroke occurred in 17 patients (7.5\%) with LSA coverage and concomitant revascularization vs 29 patients $(4.4 \%)$ with LSA coverage and no revascularization (Table 4 ). Thirty-day mortality was $5.7 \%$ and $4.4 \%$ for TEVAR with LSA coverage with vs without revascularization and the incidences of major complications were $22 \%$ and $15 \%$, respectively. After risk adjustment, compared to TEVAR without LSA coverage, TEVAR with LSA coverage and concomitant revascularization had a fourfold higher risk of stroke (OR 4.0, 95\% CI 2.2 to $7.5, \mathrm{p}<0.001$ ), and TEVAR with coverage without LSA revascularization had a 2-fold higher odds of stroke (OR 2.2, 95\% CI 1.3 to 3.8, $\mathrm{p}=0.002$; Table 4).

Of the patients undergoing isolated LSA revascularization for occlusive disease, stroke occurred in $0.5 \%$ of patients, 30 -day mortality was $0.5 \%$, and major complications occurred in $5.6 \%$ of patients.

\section{Factors Associated With Stroke Following TEVAR}

Compared to TEVAR without LSA coverage, higher odds of stroke were associated with TEVAR with LSA coverage both without (OR 2.1, 95\% CI 1.3 to $3.5, \mathrm{p}=0.005$ ) and with concomitant revascularization (OR 3.8, 95\% CI 2.1 to 7.0 , $\mathrm{p}<0.001$; Table 5). Other factors associated with stroke were preoperative dyspnea $(\mathrm{p}=0.014)$, severe preoperative renal dysfunction $(\mathrm{p}=0.049)$, and a preoperative INR $\geq 2$ $(p=0.045)$. There was no association between brachial artery access and stroke in univariate analysis or after risk adjustment (OR $0.9,95 \%$ CI 0.2 to $3.9, \mathrm{p}=0.91$ ).

\section{Discussion}

In a nationwide clinical registry, the 30-day incidence of stroke is high following TEVAR when the graft covers the origin of the LSA. In addition, both concomitant revascularization as well as no or staged revascularization were associated with higher stroke incidences compared with when the graft is deployed distally to the LSA. By comparison, stroke following isolated open LSA revascularization for occlusive disease rarely resulted in stroke.

The reported stroke incidence following TEVAR varies greatly in the literature. For TEVAR without coverage of the LSA, 2 meta-analyses reported pooled stroke incidences of $2.7 \%$ and $3.2 \%,{ }^{6,10}$ which were similar to the $2.4 \%$ stroke incidence in the current analysis. The pooled stroke incidence in meta-analyses of TEVAR with coverage of the LSA ranged between $4.7 \%$ and $7.8 \%$ without revascularization and between $4.1 \%$ and $5.8 \%$ with concomitant or staged LSA revascularization. ${ }^{5,6,10}$ Although the $4.4 \%$ stroke rate for TEVAR with LSA coverage without concomitant revascularization in our analysis was similar, the stroke rate for TEVAR with concomitant LSA revascularization $(7.5 \%)$ was higher. This difference is 
Table 5. Factors Associated With Any Postoperative Stroke Following TEVAR. ${ }^{a}$

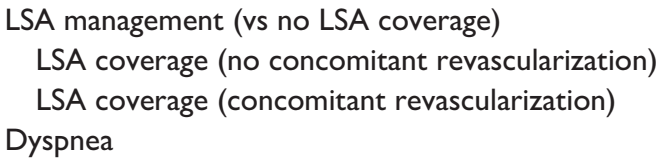

$2.1($ I .3 to 3.5$) p=0.005$
$3.8(2.1$ to 7.0$) p<0.00$ I
I.8 (I.I to 3.0$) p=0.014$
$2.0(I .0$ to 3.8$) p=0.049$
$3.6(I .0$ to 12.5$) p=0.045$

Abbreviations: INR, international normalized ratio; LSA, left subclavian artery; TEVAR, thoracic endovascular aortic repair.

${ }^{a}$ Data are presented as the odds ratio ( $95 \%$ confidence interval).

likely in part due to differences in study design, as one of the meta-analyses identified a publication bias in its analysis since larger studies reported a higher stroke incidence than smaller studies. ${ }^{6}$ In contrast, by using a national registry that includes hospitals with any volume, our analysis demonstrated that stroke might occur more frequently in TEVAR with concomitant revascularization in a real-world setting. The lack of data on prior or planned subsequent procedures limited the ability to study the difference in the stroke incidence between no or staged LSA revascularization. In addition, since patients experiencing serious adverse events during the first procedure, especially major stroke, are less likely to undergo the second stage of their procedure, the true stroke incidence may be even higher in these patients. However, this was not possible to investigate in the current study.

For TEVAR procedures that involve coverage of the LSA, the role of LSA revascularization in order to prevent stroke remains an area of debate. Using the 2005 to 2008 NSQIP dataset, Chung et $\mathrm{al}^{11}$ calculated a $5.7 \%$ overall stroke incidence for all TEVARs and determined that TEVAR with LSA coverage was associated with higher odds of stroke whereas coverage with revascularization was not. However, results from this study are difficult to compare, as it included repairs for ruptured TAA as well as concomitant open thoracic aortic procedures. In addition, the authors identified concomitant LSA revascularization only by the CPT code for combined TEVAR with revascularization. In our study using the same dataset, however, surgeons often used separate codes to register concomitant LSA revascularization, resulting in $25 \%$ of TEVAR patients with LSA coverage receiving revascularization in our study compared with $10 \%$ in the study by Chung et al. ${ }^{11}$

The current study was not able to determine the cumulative stroke incidence in TEVAR patients with staged revascularization. However, TEVAR with LSA coverage was associated with a higher stroke incidence compared to no coverage, independent of whether the LSA was concomitantly revascularized. This finding seems to be in accord with outcomes from the previously mentioned meta-analyses, which were unable to prove the benefit of prior/subsequent or concomitant LSA revascularization. Thus, evidence to support LSA revascularization solely to prevent stroke when performing TEVAR with LSA coverage is lacking. ${ }^{5,6,10}$ Our data suggest that TEVAR with coverage of the LSA, regardless of LSA management, remains associated with a higher incidence of stroke compared to TEVAR without LSA coverage. The analysis of staged vs concomitant revascularization in TEVAR with LSA coverage was beyond the scope of the current study and may be examined in future analyses.

An important consideration is that LSA revascularization with TEVAR is not performed only to prevent stroke but also to prevent ischemia of the spinal cord and left upper extremity or vertebrobasilar insufficiency, ${ }^{12,13}$ although the effectiveness of routine revascularization to prevent spinal cord ischemia also remains questionable. ${ }^{5,14,15}$ Furthermore, other more specific indications for revascularization, such as long aortic segment coverage by the stent-graft or presence of a left internal mammary artery graft, may also drive decision making. ${ }^{16}$ In addition, in specific anatomical situations, such as the presence of a dominant left vertebral artery, revascularization might still be indicated to reduce the risk of stroke following TEVAR, which could not be studied in the NSQIP. ${ }^{3}$ Therefore, the outcomes of the current study do not prove the redundancy of LSA revascularization with TEVAR in general. Rather, surgeons should be aware that revascularization alone does not prevent stroke and should take the increased stroke risk into consideration in preoperative risk assessment when performing TEVAR that requires coverage of the LSA.

Stroke was a rare complication following isolated LSA revascularization for occlusive disease. Although this procedure was performed in a different patient population, it is unlikely that the stroke incidence is higher in patients undergoing LSA revascularization for aneurysm or dissection, as they should be less likely to have atherosclerosis of the common carotid and/or subclavian arteries. The question of the etiology of strokes following TEVAR is complex. Given that concomitant revascularization does not prevent stroke and the stroke incidence is low following isolated revascularization in our study, hypoperfusion of the LSA is likely not the sole mechanism behind the occurrence of stroke. Previous studies demonstrated that aortic arch atheroma was independently associated with stroke, raising the question of whether the embolic events from 
arch manipulation rather than the hypoperfusion of the LSA are responsible. ${ }^{17-19}$

Our analysis also identified preoperative factors including dyspnea, severe renal dysfunction, and an INR $\geq 2$ as associated with a higher incidence of stroke. Associations between perioperative stroke in TEVAR patients and renal dysfunction, female sex, and prior stroke have been demonstrated previously. ${ }^{13,20}$ Our study did not demonstrate an association between stroke and sex, although it included over 7 times more female patients than the prior study. The impact of prior stroke and coronary artery disease could not be examined, as the latter variable is no longer captured by NSQIP. Elevated preoperative INR $>2$ and preoperative dyspnea might be surrogate markers for other diseases, such as atrial fibrillation or more advanced congestive heart failure or COPD. These other diseases and details of symptom severity, with the addition other previously described factors associated with stroke, such as aortic arch atheroma at the level of the branches, were not available in NSQIP. ${ }^{17}$ While it remains unclear how the results from the multivariable models would have differed with the inclusion of these variables, our data still indicate that patients presenting with these comorbidities are at higher risk of stroke. The rate of brachial artery access was low, and there was no association with perioperative stroke. This study was not able to elucidate the indication of brachial access, and therefore the impact of fenestrations, chimney, or occlusion of the LSA remains the subject to further investigation.

\section{Limitations}

The current study should be interpreted within the context of its design and has several limitations. First, the NSQIP does not provide further information on etiology or nature of stroke, such as territory affected or etiology, which would be relevant in determining the origin of strokes and the association with INR. Second, NSQIP does not capture spinal cord ischemia as an outcome, which could be relevant in the decision to revascularize the LSA. Third, no assessment was made of whether endovascular LSA revascularization techniques, such as chimney or fenestrated/branched devices, ${ }^{21,22}$ were used. However, based on the low proportion of patients with brachial artery access, it is likely that these novel strategies were in the early stages and not used widely during the data collection period. Similarly, there was no way to determine whether patients underwent LSA embolization. However, the stroke risks should be comparable between LSA occlusion by the stent-graft or embolotherapy to prevent type II endoleaks, as both lead to obstructed LSA outflow. ${ }^{23}$

Furthermore, it was unknown whether some patients had penetrating aortic ulcers, although the primary indication for each repair was verified as TAA or dissection. Lastly, while all ruptured and emergent procedures were excluded, it was not possible to verify whether some nonemergent patients were symptomatic.

\section{Conclusion}

Stroke after TEVAR is a devastating complication that leads to perioperative death in 1 of 4 patients. In a realworld setting, stroke occurs more frequently following TEVAR with coverage of the LSA regardless of the LSA management strategy, while isolated LSA revascularization rarely results in stroke. These findings question the effectiveness of routine LSA revascularization to prevent stroke after TEVAR with LSA coverage. LSA revascularization may still be warranted for specific indications, such as to prevent spinal cord ischemia or in the case of a dominant left vertebral artery.

\section{Authors' Note}

This study was presented at the 47th Annual Symposium of the Society for Clinical Vascular Surgery (March 16-19, 2019; Boca Raton, FL, USA) and the 33rd Annual Meeting of the European Society for Vascular Surgery (June 24-27, 2019; Hamburg, Germany).

\section{Declaration of Conflicting Interests}

The author(s) declared the following potential conflicts of interest with respect to the research, authorship, and/or publication of this article: Hence J. M. Verhagen is a consultant for Medtronic, W.L. Gore \& Associates, Endologix, Abbott, and Arsenal AAA. Marc L. Schermerhorn is a consultant for Abbott Vascular, Cook Medical, Endologix, Medtronic, and Silk Road.

\section{Funding}

The author(s) disclosed receipt of the following financial support for the research, authorship, and/or publication of this article: Kirsten Dansey is supported by the Harvard-Longwood Research Training in Vascular Surgery NIH T32 Grant 5T32HL007734-22.

\section{ORCID iDs}

Rens R. B. Varkevisser iD https://orcid.org/0000-0002-1998-5159 Marc L. Schermerhorn (iD https://orcid.org/0000-0002-4278-964X

\section{Supplemental Material}

Supplemental material for this article is available online.

\section{References}

1. Walsh SR, Tang TY, Sadat U, et al. Endovascular stenting versus open surgery for thoracic aortic disease: Systematic review and meta-analysis of perioperative results. J Vasc Surg. 2008;47:1094-1098.

2. Li F-R, Wu X, Yuan J, et al. Comparison of thoracic endovascular aortic repair, open surgery and best medical treatment for type B aortic dissection: A meta-analysis. Int $J$ Cardiol. 2018;250:240-246. 
3. Feezor RJ, Martin TD, Hess PJ, et al. Risk factors for perioperative stroke during thoracic endovascular aortic repairs (TEVAR). $J$ Endovasc Ther. 2007;14:568-573.

4. Matsumura JS, Lee WA, Mitchell RS, et al. The Society for Vascular Surgery Practice Guidelines: management of the left subclavian artery with thoracic endovascular aortic repair. $J$ Vasc Surg. 2009;50:1155-1158.

5. Hajibandeh S, Hajibandeh S, Antoniou SA, et al. Metaanalysis of left subclavian artery coverage with and without revascularization in thoracic endovascular aortic repair. $J$ Endovasc Ther. 2016;23:634-641.

6. von Allmen RS, Gahl B, Powell JT. Editor's choice. Incidence of stroke following thoracic endovascular aortic repair for descending aortic aneurysm: a systematic review of the literature with meta-analysis. Eur J Vasc Endovasc Surg. 2017;53: 176-184.

7. Davis CL, Pierce JR, Henderson W, et al. Assessment of the reliability of data collected for the Department of Veterans Affairs National Surgical Quality Improvement Program. J Am Coll Surg. 2007;204:550-560.

8. Shiloach M, Frencher SK, Steeger JE, et al. Toward robust information: data quality and inter-rater reliability in the American College of Surgeons National Surgical Quality Improvement Program. J Am Coll Surg. 2010;210:6-16.

9. Levey AS, Stevens LA, Schmid CH, et al. A new equation to estimate glomerular filtration rate. Ann Intern Med. 2009;150: 604-612.

10. Cooper DG, Walsh SR, Sadat U, et al. Neurological complications after left subclavian artery coverage during thoracic endovascular aortic repair: a systematic review and metaanalysis. J Vasc Surg. 2009;49:1594-1601.

11. Chung J, Kasirajan K, Veeraswamy RK, et al. Left subclavian artery coverage during thoracic endovascular aortic repair and risk of perioperative stroke or death. J Vasc Surg. 2011;54: 979-984.

12. Murphy EH, Stanley GA, Ilves M, et al. Thoracic endovascular repair (TEVAR) in the management of aortic arch pathology. Ann Vasc Surg. 2012;26:55-66.

13. Buth J, Harris PL, Hobo R, et al. Neurologic complications associated with endovascular repair of thoracic aortic pathology:
Incidence and risk factors. A study from the European Collaborators on Stent/Graft Techniques for Aortic Aneurysm Repair (EUROSTAR) Registry. J Vasc Surg. 2007;46: 1103-1111.

14. Maldonado TS, Dexter D, Rockman CB, et al. Left subclavian artery coverage during thoracic endovascular aortic aneurysm repair does not mandate revascularization. J Vasc Surg. 2013;57:116-124.

15. Lee TC, Andersen ND, Williams JB, et al. Results with a selective revascularization strategy for left subclavian artery coverage during thoracic endovascular aortic repair. Ann Thorac Surg. 2011;92:97-103.

16. Drinkwater SL, Goebells A, Haydar A, et al. The incidence of spinal cord ischaemia following thoracic and thoracoabdominal aortic endovascular intervention. Eur J Vasc Endovasc Surg. 2010;40:729-735.

17. Kotelis D, Bischoff MS, Jobst B, et al. Morphological risk factors of stroke during thoracic endovascular aortic repair. Langenbecks Arch Surg. 2012;397:1267-1273.

18. Amarenco P, Cohen A, Tzourio C, et al. Atherosclerotic disease of the aortic arch and the risk of ischemic stroke. $N$ Engl J Med. 1994;331:1474-1479.

19. Bismuth J, Garami Z, Anaya-Ayala JE, et al. Transcranial Doppler findings during thoracic endovascular aortic repair. J Vasc Surg. 2011;54:364-369.

20. Ullery BW, McGarvey M, Cheung AT, et al. Vascular distribution of stroke and its relationship to perioperative mortality and neurologic outcome after thoracic endovascular aortic repair. J Vasc Surg. 2012;56:1510-1517.

21. Hogendoorn W, Schlösser FJV, Moll FL, et al. Thoracic endovascular aortic repair with the chimney graft technique. $J$ Vasc Surg. 2013;58:502-511.

22. Redlinger RE, Ahanchi SS, Panneton JM. In situ laser fenestration during emergent thoracic endovascular aortic repair is an effective method for left subclavian artery revascularization. J Vasc Surg. 2013;58:1171-1177.

23. Peterson MD, Wheatley GH, Kpodonu J, et al. Treatment of type II endoleaks associated with left subclavian artery coverage during thoracic aortic stent grafting. J Thorac Cardiovasc Surg. 2008;136:1193-1199. 